\title{
Proposal of computer aided process planning of parts machining using workholding devices
}

\author{
Wataru KOMATSU*, Yuki INOUE* and Keiichi NAKAMOTO* \\ *Department of Mechanical Systems Engineering \\ Tokyo University of Agriculture and Technology, Japan \\ 2 Nakamachi, Koganei-shi, Tokyo 184-8788, Japan \\ E-mail: nakamoto@cc.tuat.ac.jp
}

Received: 14 June 2019; Accepted: 17 September 2019

\begin{abstract}
High efficiency machining achieved by shortening preparation time has attracted attention for its many advantages in the field of machining. In order to reduce preparation time, computer-aided process planning (CAPP) is expected to automatically determine machining process parameters such as machining sequence, cutting tools, and cutting conditions. In the author's previous study, novel machining features are proposed to derive machining process parameters from specific geometric shape patterns of removal volume during a parts machining operation. It is also confirmed that the machining features are recognized by deciding the machining sequence for each machining primitive that is first obtained from the removal volume. However, workholding devices have not been considered to determine the machining sequence or to recognize the machining features in parts machining operation. Workholding devices are generally indispensable for parts machining and are practically important to determine machining sequences. Therefore, this study aims to develop a CAPP system for parts machining considering the use of workholding devices. Two general workholding devices, a machine vise and strap clamps, are assumed for various parts machining operations. Under the limitations of workholding devices, workpiece fixing positions are calculated before the machining features are recognized through allocating the machining sequence. Moreover, the number of fixing changes and the machining time are estimated according to both the workholding device being used and the workpiece fixing positions. Case studies are conducted to confirm the usefulness of the developed CAPP system considering the use of workholding devices. The results reveal that fixing positions are obtained properly for various combinations of workpieces and workholding devices. Furthermore, it is confirmed that the CAPP system has the potential to be effective for automatically determining the machining process parameters of parts machining.
\end{abstract}

Keywords : Process planning, Parts machining, Workholding device, Machining sequence, Machining feature

\section{Introduction}

Manufacturing in recent years has tended toward high-mix, low-volume production. Therefore, high efficiency machining by shortening preparation time has attracted attention for its many advantages in the field of machining. Computer support technologies, computer aided design (CAD), computer aided manufacturing (CAM), and computeraided process planning (CAPP), which bridges CAD and CAM systems, are indispensable for high-mix, low-volume production. Among these technologies, CAM systems must minimize the effort of numerical control (NC) programming in order to realize high-efficiency parts machining. However, a commercial CAM system commonly takes a long time to manually allocate cutting tools, cutting conditions and so on. As a result, $\mathrm{NC}$ programming is strongly expected to reduce preparation time in CAM systems by using the output data of CAPP systems.

In a CAPP system, it is necessary to automatically determine machining process parameters such as machining sequences, cutting tools, and cutting conditions, according to CAD models of target and workpiece shapes. Recognition 
of machining features that are specific geometric shape patterns is known as a key to determining machining process parameters automatically. Various methods of recognizing machining features have been proposed based on either target shape (Wang et al., 2010) (El-Mehalawi and Miller, 2003a, 2003b) or removal volume (Dwijayanti and Aoyama, 2014) (Morinaga et al., 2014) (Nishida et al., 2017) (Nishida et al., 2018). On the other hand, the authors also have proposed novel machining features that are recognized by dividing the removal volume using the faces contacting the target shape (Ueno and Nakamoto, 2015). Furthermore, a CAPP system is developed by referring the recognized machining features for parts machining (Inoue and Nakamoto, 2017).

However, the fixing limitations of a workpiece have not been considered to recognize the machining features in these methods. Workholding devices are practically indispensable for parts machining and are generally important for determining the machining sequence. Though numerical simulations of workpiece deformation (Ratchev et al., 2004) (Siebenaler and Melkote, 2006), and the modeling of workholding states (Teramoto, 2017) have been studied, those studies did not involve process planning. Therefore, this study aims to develop a CAPP system of parts machining considering the use of workholding devices. Two general workholding methods, a machine vise and strap clamps, are assumed for various parts machining operations. Under the limitations of workholding devices, the suitable workpiece fixing positions are calculated before the recognition of machining features through allocating the machining sequence. Moreover, the number of fixing changes and the machining time are estimated based on the combinations of the workholding devices used and the workpiece fixed positions. Case studies are conducted to confirm the usefulness of the developed CAPP system considering the use of workholding devices. The results show that fixing positions are obtained properly for various workpieces and workholding devices. Furthermore, it is confirmed that the CAPP system has a potential to be effective for automatically determining the machining process parameters in parts machining.

\section{Recognition of machining features}

Recognition of machining features is a key to determining machining process parameters such as machining sequences, cutting tools, and cutting conditions. Machining features are characteristic shape patterns that can identify specific machining processes. In the author's previous study, novel machining features are proposed and their recognition method is devised (Ueno and Nakamoto, 2015). In this method, the removal volume is first extracted as the difference of the workpiece and target shapes. Then, the removal volume is divided into simple shapes called machining primitives. In order to obtain machining primitives, boundary faces where the removal volume and the target shape come into contact are detected. Additionally, the boundary faces are extended to the inside of the removal volume so that machining primitives are obtained by using the extended boundary faces to divide the removal volume. The machining sequence is allocated to each machining primitive by following several specific rules, and the machining primitives are classified to recognize their machining features with respect to geometric information such as the edges and generated surfaces where the machining primitive and the target shape come into contact. Figure 1 shows an example result of recognized machining features in the author's previous study.

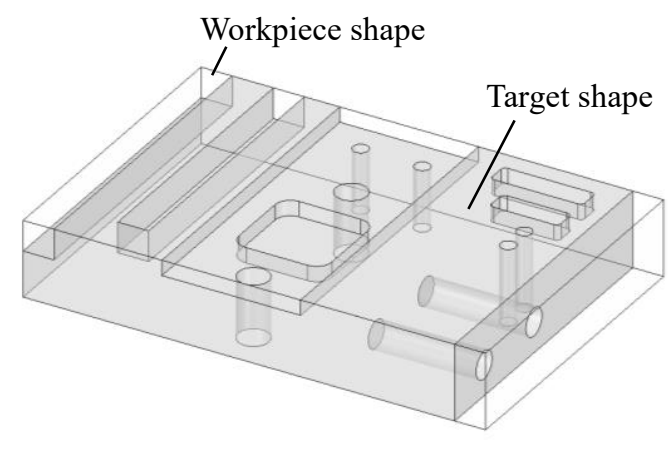

Target and workpiece shapes

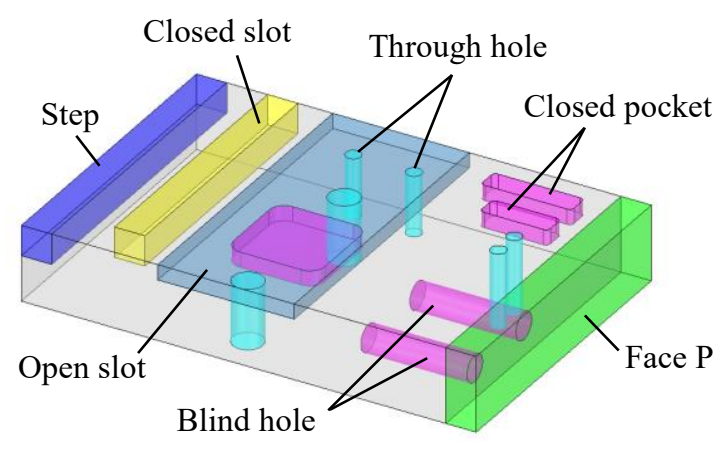

Recognized machining features

Fig. 1 Recognition of machining features in the author's previous study (Inoue and Nakamoto, 2017) 


\section{Developed CAPP system}

In this study, a CAPP system is developed for parts machining considering the use of workholding devices. In the developed CAPP system, both CAD models of the workpiece and the target shapes, the specifications of workholding devices and toolings are first inputted. Then, several kinds of workpiece fixing positions are calculated according to the workholding device and the previously proposed machining features are recognized through allocation of the machining sequence automatically as shown in Fig. 2. Machining process parameters are finally determined based on the recognized machining features, and a suitable workholding device can be selected by referring to the number of fixing changes and the machining time.

A rectangular solid is treated as the workpiece shape in this study. Two general workholding devices, a machine vise and strap clamps, are assumed to fix the workpiece for parts machining operations on a three-axis control machining center. The API of Simple Modeler (AIKOKU ALPHA Corp.) with C\# is used to develop the CAPP system.

\subsection{Workpiece fixing by using a machine vise}

This section describes the procedure to decide on workpiece fixing positions and to recognize the machining features in the case of the use of a machine vise. In this case, jaw depth, jaw width, jaw opening, and the minimum fixing area are required as workholding device specifications, as shown in Fig. 3. Appropriate fixing positions are calculated by considering the position relation between the workpiece shape and the removal volume. First, the bottom face of the fixed workpiece is detected. There are two types, "Type A" and "Type B", to detect the bottom face. Accordingly, the fixing positions and the machining sequence are decided according to the type, respectively.

In Type A, the workpiece is sequentially divided by XY, YZ, or ZX plane at the coordinate origin through the workpiece center as shown in Fig. 4. Then, each removal volume of the divided workpiece is calculated. The upper face of the largest removal volume of the divided workpiece is detected as the top face as shown in Fig. 5. Finally, the face

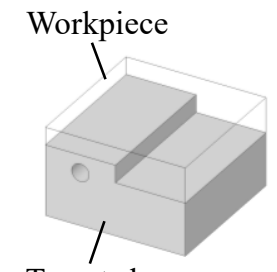

Target shape

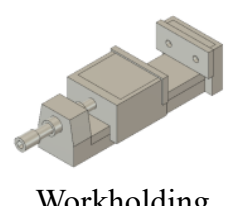

Workholding devices

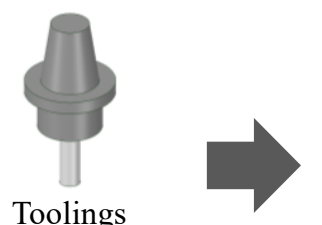

Toolings

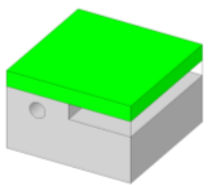

Primitive A

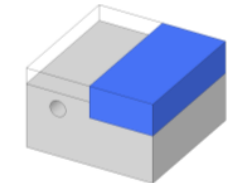

Primitive B

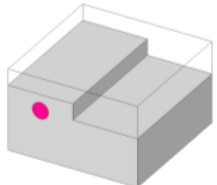

Primitive C

(1) Input of workpiece and target shapes, specifications of workholding devices and toolings

(2) Obtainment of machining primitives

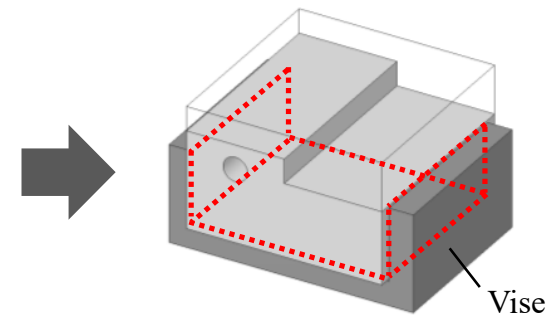

(3) Decision on workpiece fixing position

Seq. 3 : Machining feature c

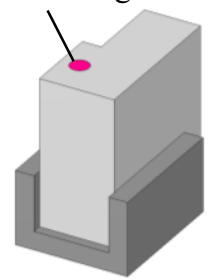

(5) Change in fixing position and re-allocation of machining sequence

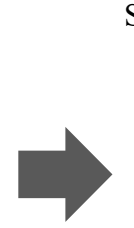

Seq. 1 : Machining feature a
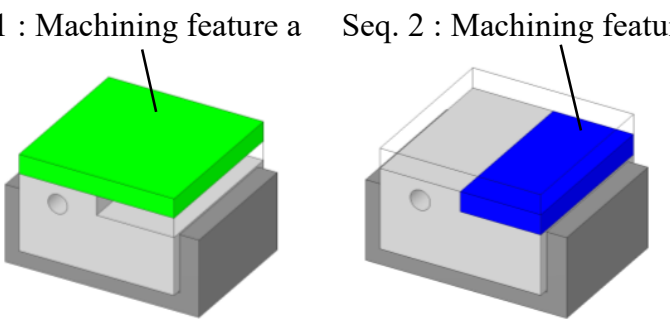

(4) Allocation of machining sequence and recognition of machining feature

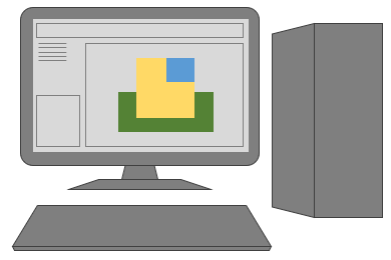

(6) Determination of machining process parameters

Fig. 2 Flow of developed CAPP system for parts machining using workholding device 


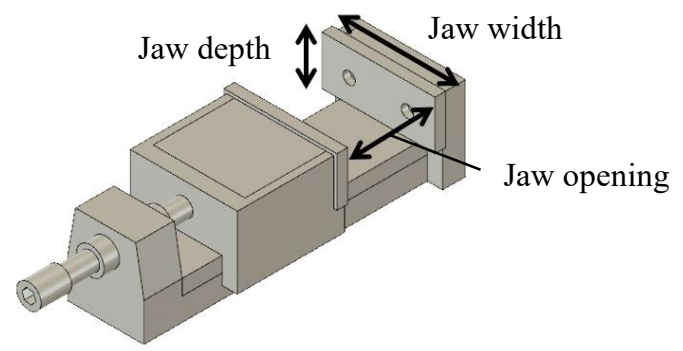

Fig. 3 Required specifications of machine vise

opposite the top face is detected as the bottom face of Type A. In Type B, the face contacting the largest number of machining primitives is detected as the top face of the chucked workpiece because this face is assumed to contain the largest machining area. An example to the detected top face is shown in Fig. 6. Then, the face opposite the top face is detected as the bottom face of Type B.

After the bottom face is decided, the side faces of the fixed workpiece are selected. In order to select side faces that contact the jaw of the machine vise, the distance from the bottom face to the removal volume is calculated at each sideface candidate. Then, a pair of faces having the longest distance are selected as the side faces. An example of side-face selection is shown in Fig. 7.

Figure 8 shows the contact length in the height direction between the side faces and the machine vise jaw. The contact length is originally set as the minimum distance from the bottom face to the removal volume. If this length is greater than

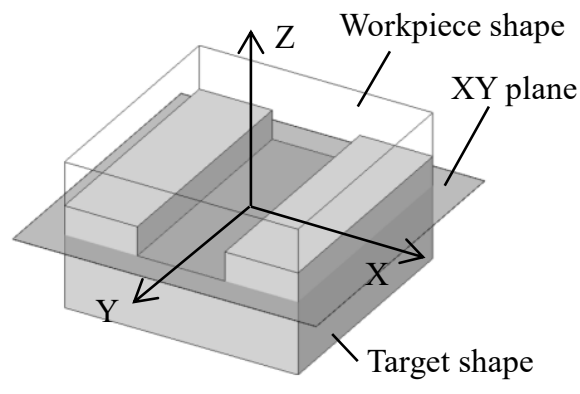

(a) XY plane

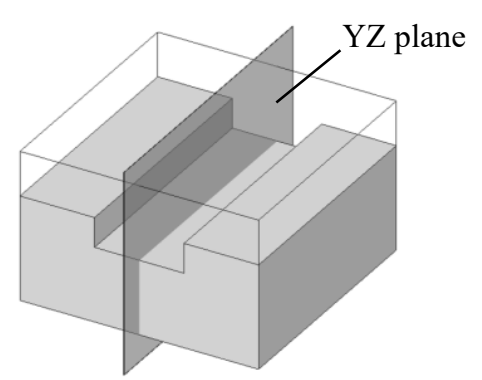

(b) YZ plane

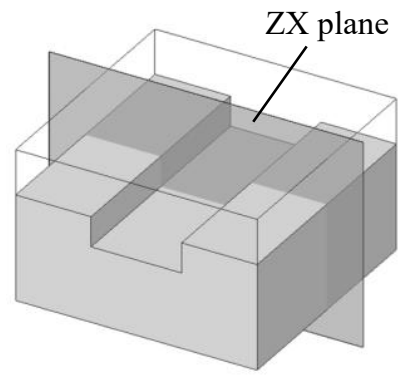

(c) ZX plane

Fig. 4 Workpiece divided by planes to detect the bottom face of chucked workpiece

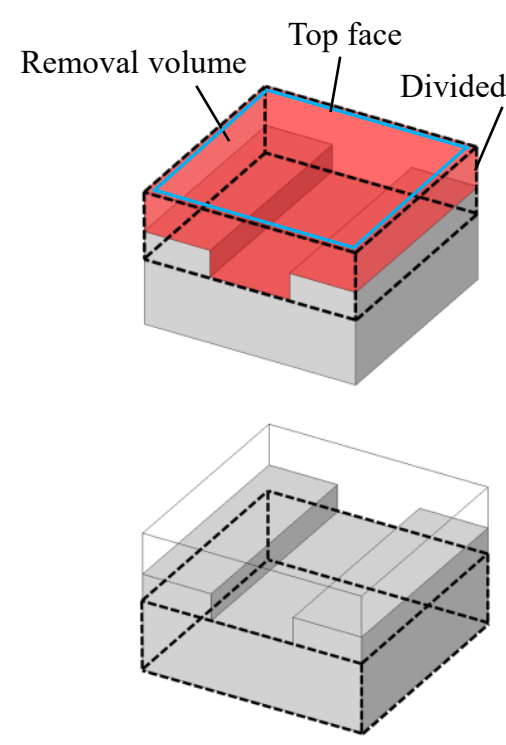

(a) XY plane

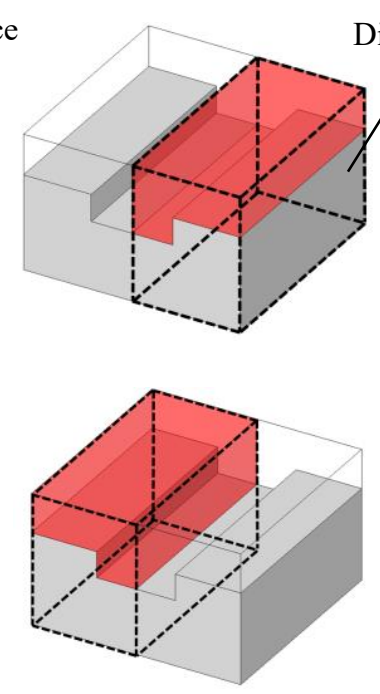

(b) YZ plane

Divided workpiece

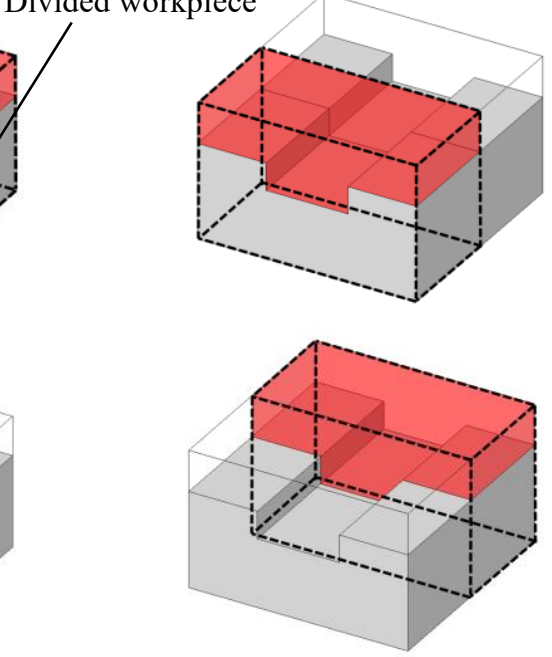

(c) ZX plane

Fig. 5 Removal volume of divided workpiece and top face having the largest volume in Type A 


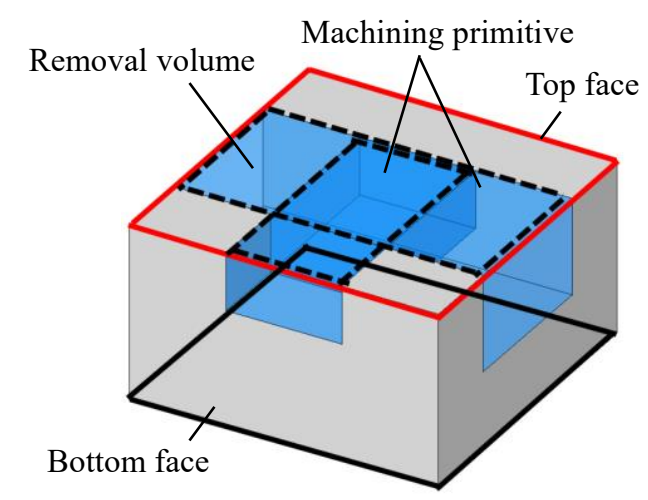

Fig. 6 Detected top face depending on the number of machining primitives

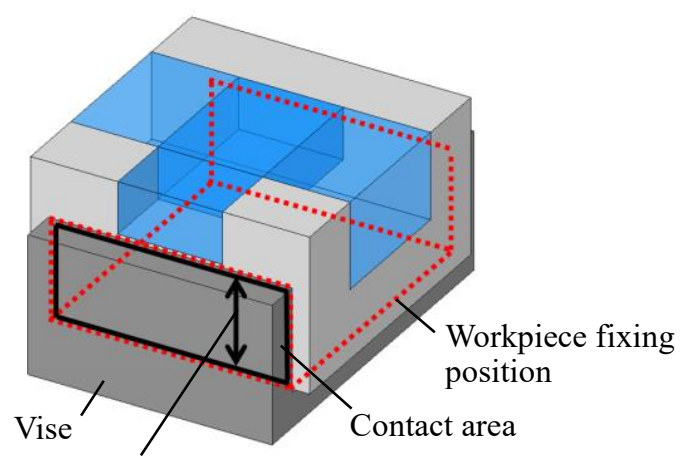

Contact length in height direction

Fig. 8 Contact length and area between side face and jaw of machine vise

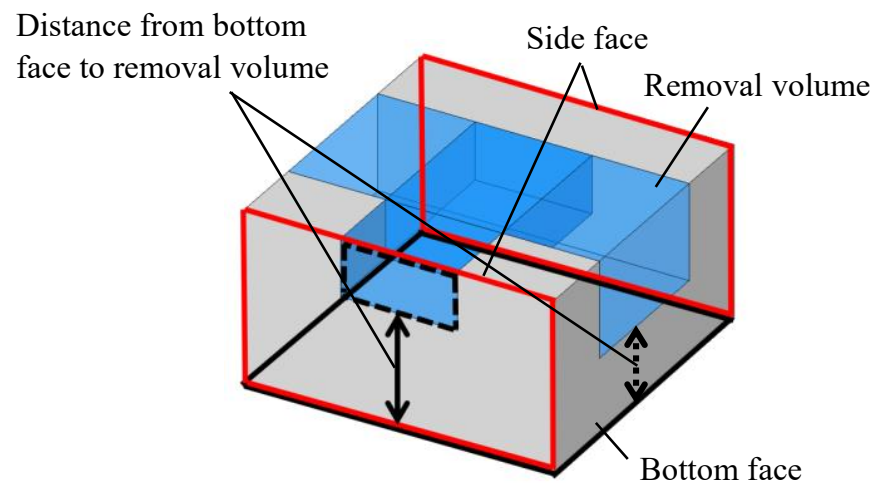

Fig. 7 Selected side faces by comparing the distances from bottom face to removal volume

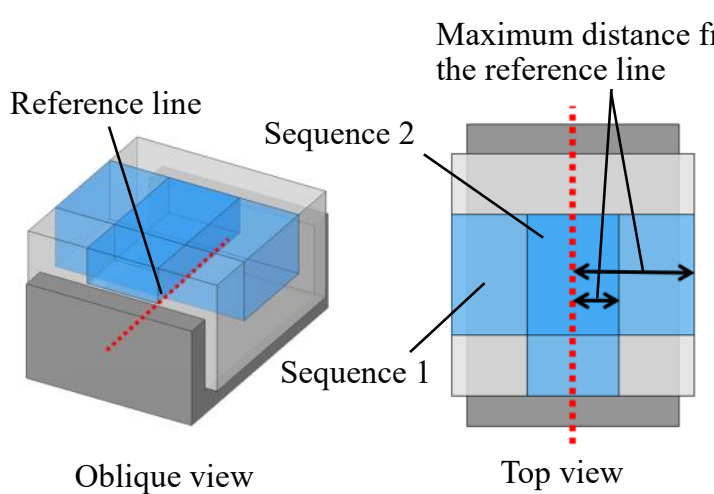

Fig. 9 Reference line passing through center of machine vise jaw for allocating machining sequence

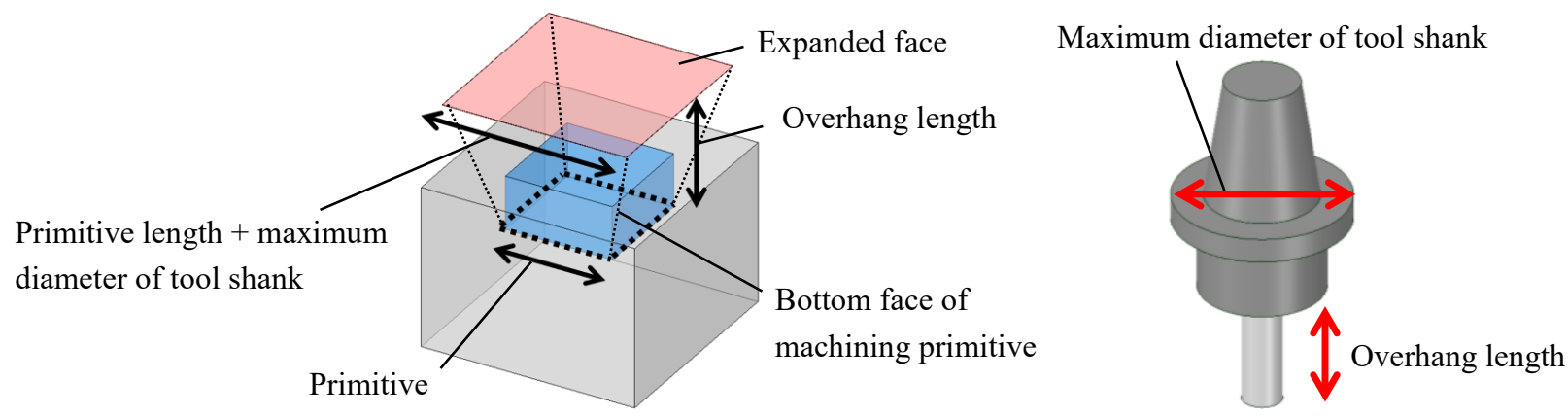

Fig. 10 Expanded face for checking tooling interference

the vise's jaw depth, the contact length is changed to the jaw depth. Furthermore, the contact area between the side face and the machine vise jaw is calculated. If the contact area is larger than the minimum fixing area, the contact length in the height direction is adopted and the workpiece fixing position is decided. Otherwise, the machining primitive that is the closest to the bottom face is subtracted from the removal volume to increase the contact length. The contact length is set as the minimum distance again, and this procedure is repeated until the suitable contact area is ensured to exceed the minimum fixing area. If the contact length cannot be set while all machining primitives are subtracted, the side faces or the bottom face would be changed.

After the fixing position is decided, the machining sequence is allocated to each machining primitive in descending order of the maximum distance from the reference line passing through the center of the machine vise jaw, as shown in Fig. 9. When the machining sequence is allocated, tooling interference is simply checked against the workpiece and a machine vise. In order to detect the tooling interference, the maximum diameter of the tool shank and the overhang length are derived from the tooling specifications. Then, the outline of the bottom face of the machining primitive is expanded 
by the maximum diameter at each edge. The expanded face is placed at the plane where the distance from the bottom face becomes the overhang length of tooling, as shown in Fig. 10. If there is a collision between the expanded face and the workpiece or the machine vise, tooling interference is assumed to happen and the machining sequence is not allocated to this machining primitive.

If there remains a machining primitive that machining sequence cannot be allocated, the fixing position is changed by detecting a new bottom face by using the updated removal volume according to one of the previously mentioned types, Type A or Type B, again. Then, the new side faces and their contact lengths are calculated similarly, the new fixing position is decided and the machining sequence is allocated continuously. This procedure is repeated until the machining sequence is allocated to all of the machining primitives, or the machining sequence could not be allocated to any machining primitives without the change in the workholding device. Finally, machining features are recognized for each machining primitive.

\subsection{Workpiece fixing by using strap clamps}

This section describes the procedure to decide the workpiece fixing position and to recognize machining features in a case where strap clamps are used. In this case, the clamp width, length, and thickness, the contact length of the workpiece, and the acceptable workpiece height are required specifications, as shown in Fig. 11.

Appropriate fixing positions are calculated by considering the position relation between the workpiece shape and the removal volume. Two to four strap clamps are used to fix a workpiece. The face that contact the largest number of machining primitives is first detected as the top face of the fixed workpiece. Then, an arbitrary edge consisting of the top face is selected (Fig. 12(i)). At the selected edge, the area where a clamp can be located is set by referring to the contact length of the workpiece (Fig. 12(ii)). Additionally, machining primitives contained in the area are extracted (Fig. 12(iii)). Line segments are also generated at the edge so as to avoid the overlap between the machining primitives and the contact area (Fig. 12(iv)). As a result, a tentative fixing position is decided at the center of the longest line segment (Fig. 12(v)). If the tentatively decided clamp contacts a machining primitive, the largest volume of machining primitive in the area is

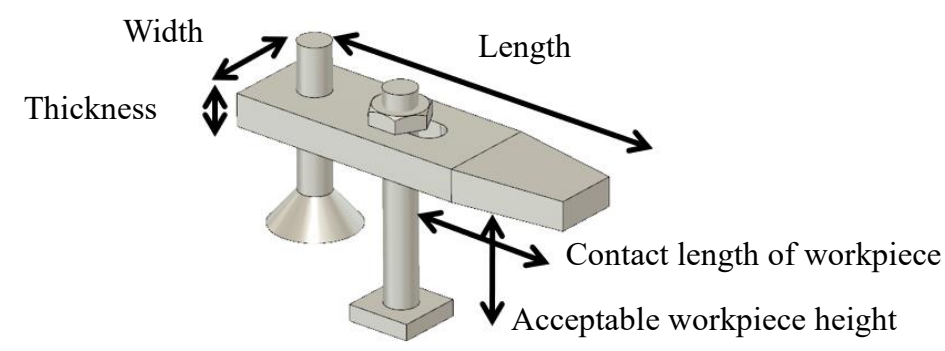

Fig. 11 Required specifications of strap clamps

(i)

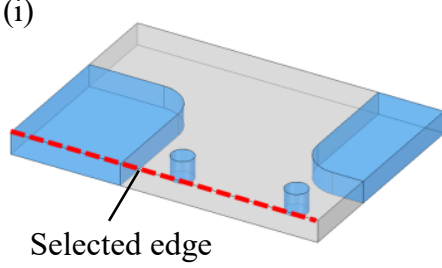

(iv)

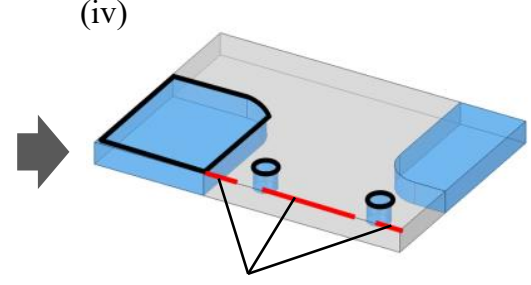

Generated line segments (ii)

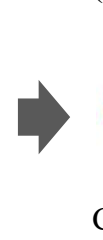

Created area

(v)

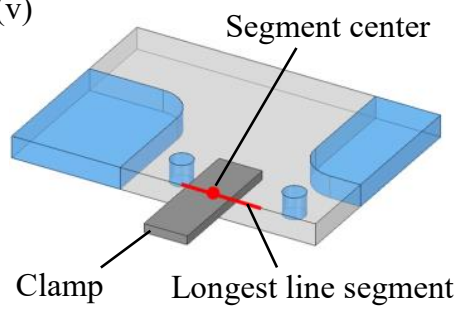

(iii)

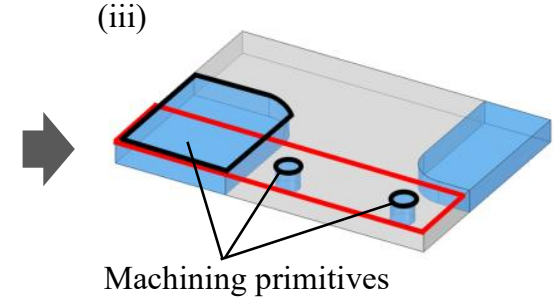

(vi)

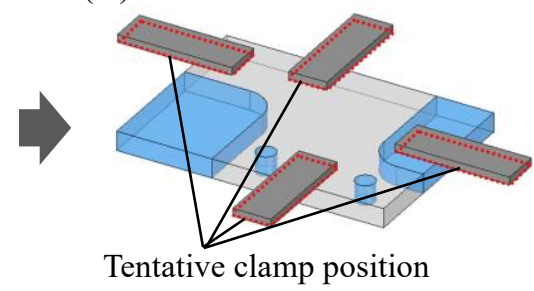

Fig. 12 Procedure to decide workpiece fixing position by using strap clamps 


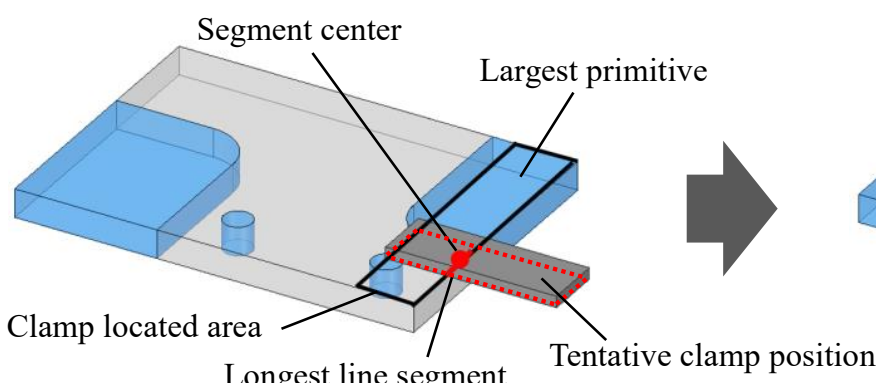

Longest line segment

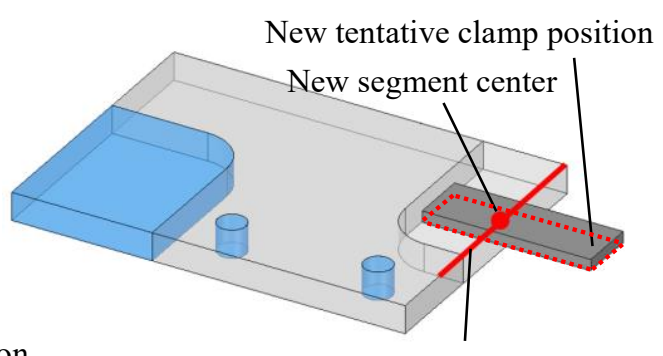

New longest line segment

Fig.13 Change in tentative clamp position contacting machining primitive

removed and the tentative clamp position is changed according to the new longest line segment, as shown in Fig. 13. This procedure is sequentially conducted at each edge (Fig. 12(vi)). As a result, four tentative clamp positions are decided on the workpiece. Among these four tentative clamp positions, two to four tentative clamps are adopted as the fixing positions. The number of clamps used is decided as follows.

- When two or more tentatively decided clamps don't contact any machining primitives, all of them are adopted as the fixing positions.

- When only one tentatively decided clamp doesn't contact any machining primitives, the clamp position and the opposite position are adopted as the fixing positions. Here, the contact between the clamp and the machining primitives is ignored at the opposite position.

- When all tentatively decided clamps contact machining primitives, one clamp position is adopted randomly. Then, the opposite position is also adopted as the fixing position. The contact between clamps and machining primitives is ignored in both positions.

After the fixing positions are decided, a machining sequence is allocated to each machining primitive in descending order of the distance from the workpiece center. When the machining sequence is allocated, tooling interference is simply checked similar to the case where a machine vise is used.

If there remains a machining primitive that does not allow the allocation of a machining sequence, the fixing position is changed by detecting a new top face. A face contacting the largest number of remaining machining primitives is selected as the new top face. Then, new fixing positions are decided similarly and the machining sequence is allocated continuously. This procedure is repeated until the machining sequence is allocated to whole machining primitives, or the machining sequence could not be allocated to any machining primitives without changing the workholding device. Finally, machining features are recognized for each machining primitive.

\subsection{Workpiece fixing by both machine vise and strap clamps}

This section, a case where both a machine vise and strap clamps are used for workpiece fixing is described. In this case, two kinds of fixing methods are used: the "vise-preceding method" and the "clamp-preceding method".

In the vise-preceding method, the fixing position is first decided by using a machine vise, and machining sequence is allocated according to the procedure described in 3.1. Then, if a machining primitive with an unallocated machining sequence remains, strap clamps are used instead of a vise. The fixing position and the machining sequence are decided for the remaining machining primitives according to the procedure described in 3.2.

In the clamp-preceding method, the order of the use of workholding devices is opposite that of the vise-preceding method. First, the fixing position is decided by using strap clamps, and the machining sequence is allocated. Then, if the machining primitive with unallocated machining sequences remains, the vise is used instead of clamps. The fixing position and the machining sequence are decided for the remaining machining primitives.

\section{Case study}

To confirm the usefulness of the developed CAPP system, three case studies are conducted: one using a machine vise, one using strap clamps, and one using both a machine vise and strap clamps. Here, fixing positions are decided and machining features are recognized for a couple of target shapes by following the author's previously proposed method (Ueno and Nakamoto, 2015). Additionally, machining simulation is conducted by using commercial software, Fusion 360 (Autodesk Corp. ) to estimate the machining time. The workpiece material is assumed as A5052, and the largest 
diameter tool is selected for each machining feature; $\varphi 4,6,8,10$, and 25 carbide square-end mills and $\varphi 8,10$, and 12 carbide drills are assumed to be prepared. The cutting conditions used are those recommended by the tool manufacturer.

\subsection{Workpiece fixing by using a machine vise}

This section, a machine vise is assumed to be the workholding device. The target shape, workpiece shape, and specifications of the machine vise are shown in Fig. 14. The decided fixing positions and the machining features categorized as Type A or Type B are shown in Figs. 15 and 16, respectively. It is confirmed that these fixing positions and machining sequences are totally different from each other. As described in 3.1, the positional relationship between the removal volume and the workpiece is considered to decide the fixing position in Type A. Therefore, the face consisting of the largest removal volume is detected as the first top face. On the other hand, in Type B, the number of machining primitives in the face is treated to decide the fixing position. Therefore, the face containing the largest number of machining primitives is detected as the first top face. From the comparison results between Type A and Type B, the second fixing position of Type A has a smaller contact area because the machined area of the first fixing position is quite large. Machining time without fixing change time is estimated as 34 minutes 15 seconds for both Type A and Type B.

\subsection{Workpiece fixing by using strap clamps}

This section, strap clamps are assumed to be the workholding device. The target shape, workpiece shape, and clamp specifications are shown in Fig. 17. The decided fixing positions and recognized machining features are shown in Fig. 18. As shown in this figure, three strap clamps at three edges ( $a, b$, and $d$ ), are adopted to fix the workpiece. These fixing positions are decided so as to avoid contact against the machining primitives. At edge c, there is no area where a clamp can be located without making contact with against the machining primitives. Machining time without fixing change time is estimated at 7 minutes 12 seconds.
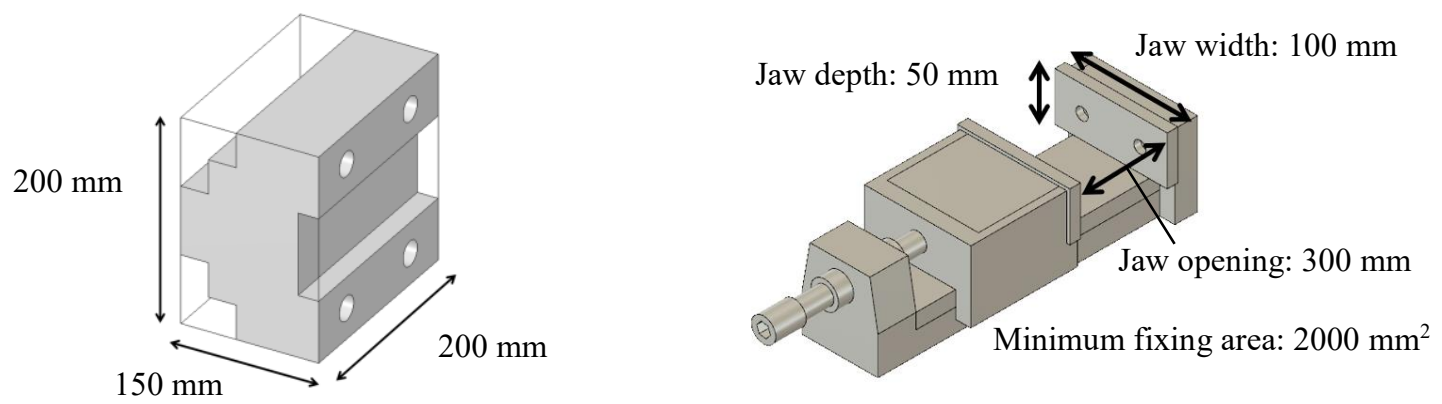

Fig. 14 Workpiece shape, target shape, and vise specifications

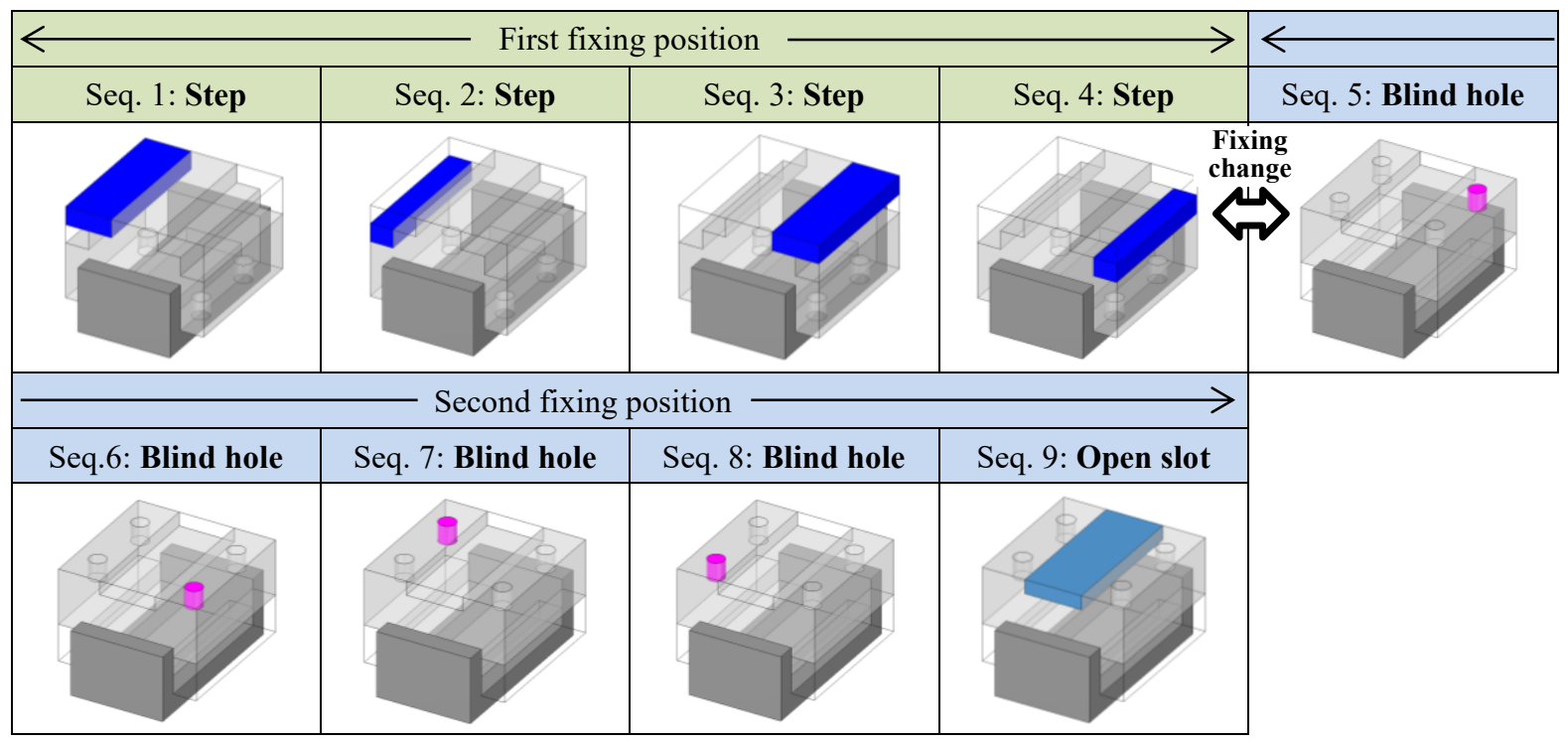

Fig. 15 Decided fixing positions and recognized machining features in Type A 


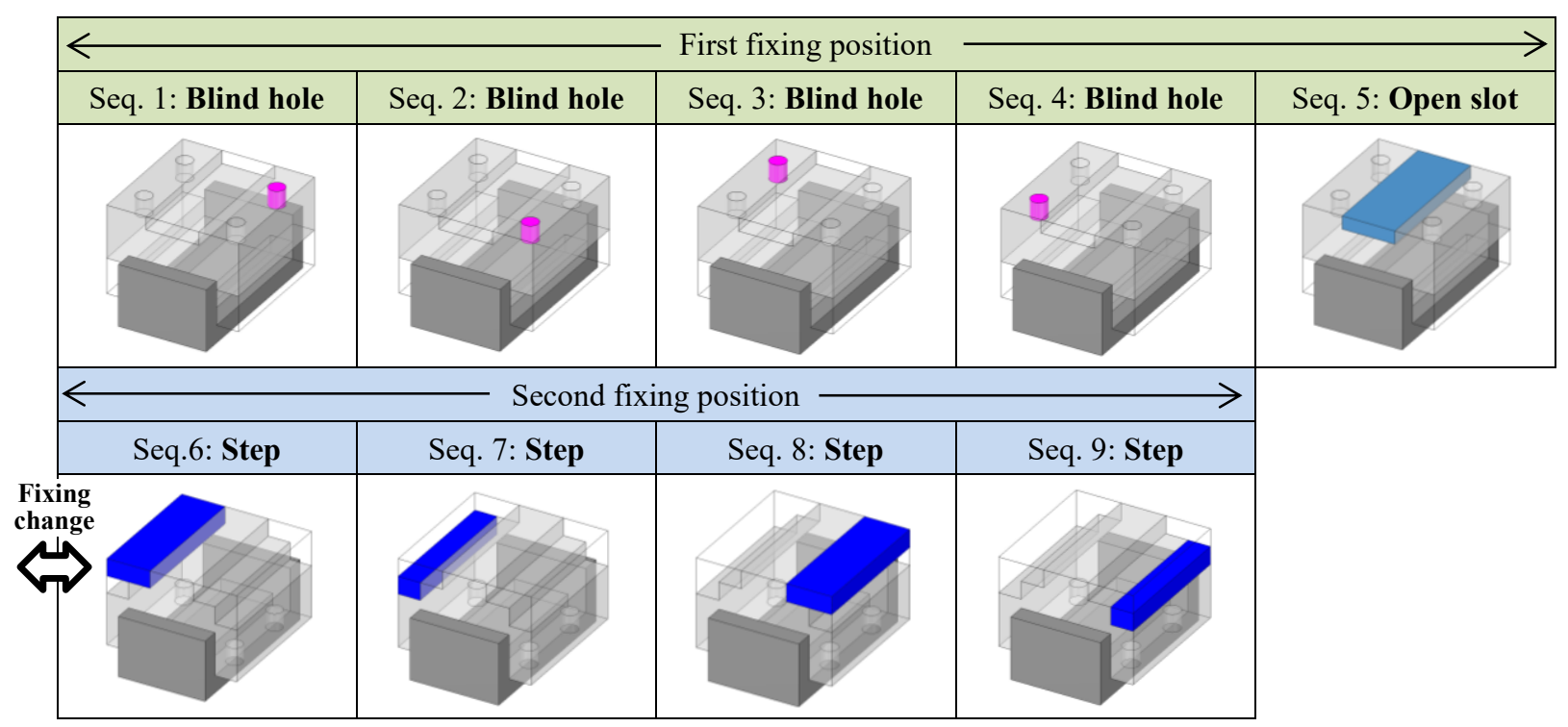

Fig. 16 Decided fixing positions and recognized machining features in Type B
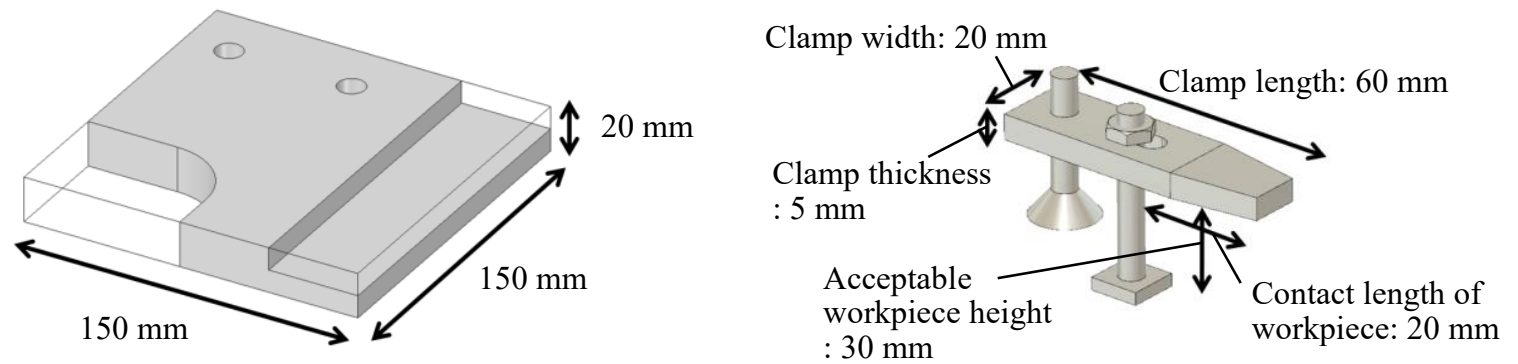

Fig. 17 Workpiece shape, target shape, and clamp specifications

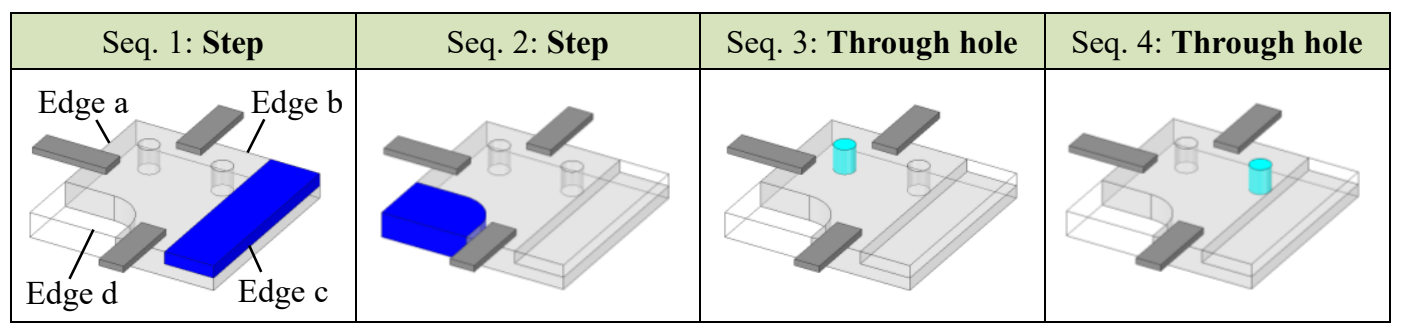

Fig. 18 Decided fixing positions and recognized machining features in the case of using strap clamps

\subsection{Workpiece fixing by both machine vise and strap clamps}

This section, both a machine vise and strap clamps are assumed to be the used workholding devices. Target shape, workpiece shape and the specifications of the machine vise and strap clamp are shown in Fig. 19. The decided fixing positions and recognized machining features according to both the vise-preceding method and the clamp-preceding method are shown in Figs. 20 and 21, respectively. It is found that workpiece fixing changes three times in the visepreceding method. On the other hand, workpiece fixing changes twice in the clamp-preceding method. Respective machining times without fixing change are estimated as 9 minutes 45 seconds in the vise-preceding method and 8 minutes 14 seconds in the clamp-preceding method.

These case studies demonstrate that the developed CAPP system enables the determination of machining process parameters of parts machining considering the use of workholding devices. Moreover, users of the developed CAPP system can obtain useful information such as the number of fixing changes and the machining time according to workholding devices. These results show that parts machining is realized based on the decided workpiece fixing positions and the recognized machining features of the developed CAPP system. 


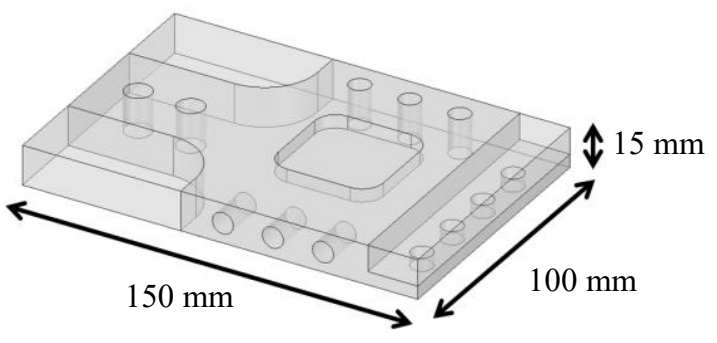

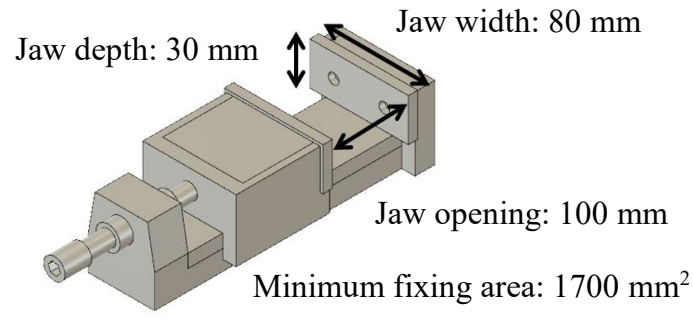

Vise specifications

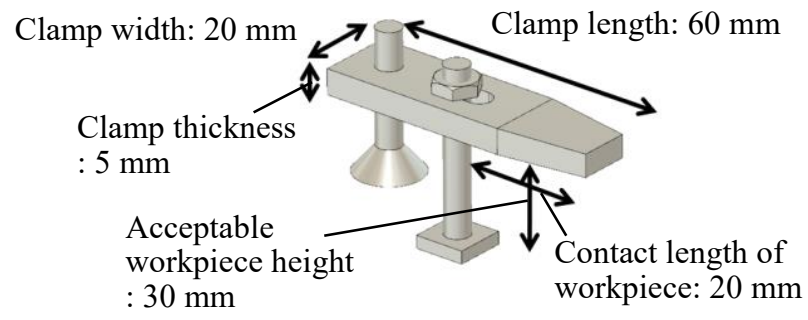

Clamp specifications

Fig. 19 Workpiece shape, target shape, and specifications of workholding devices

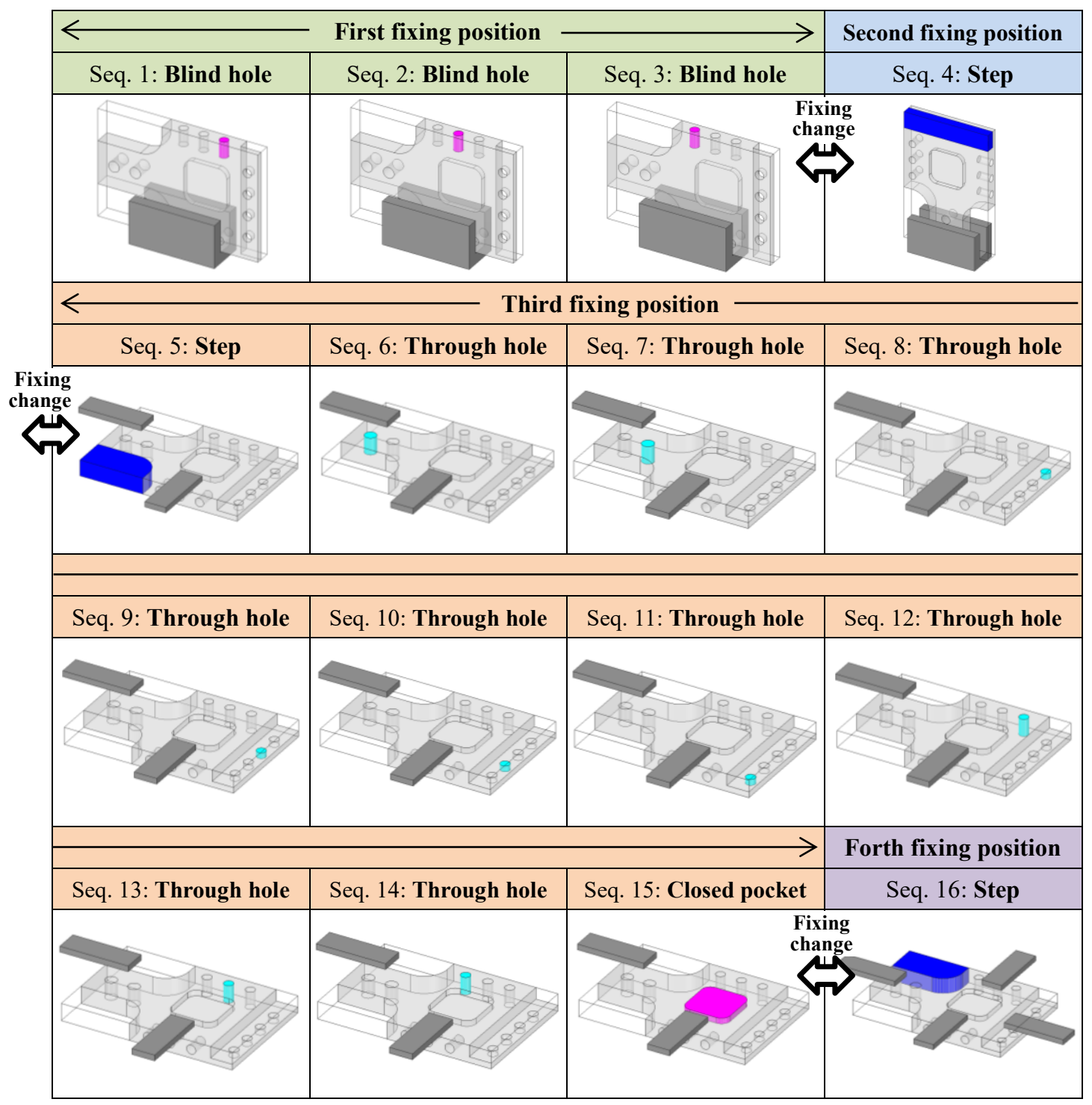

Fig. 20 Decided fixing position and recognized machining features in vise-preceding method 


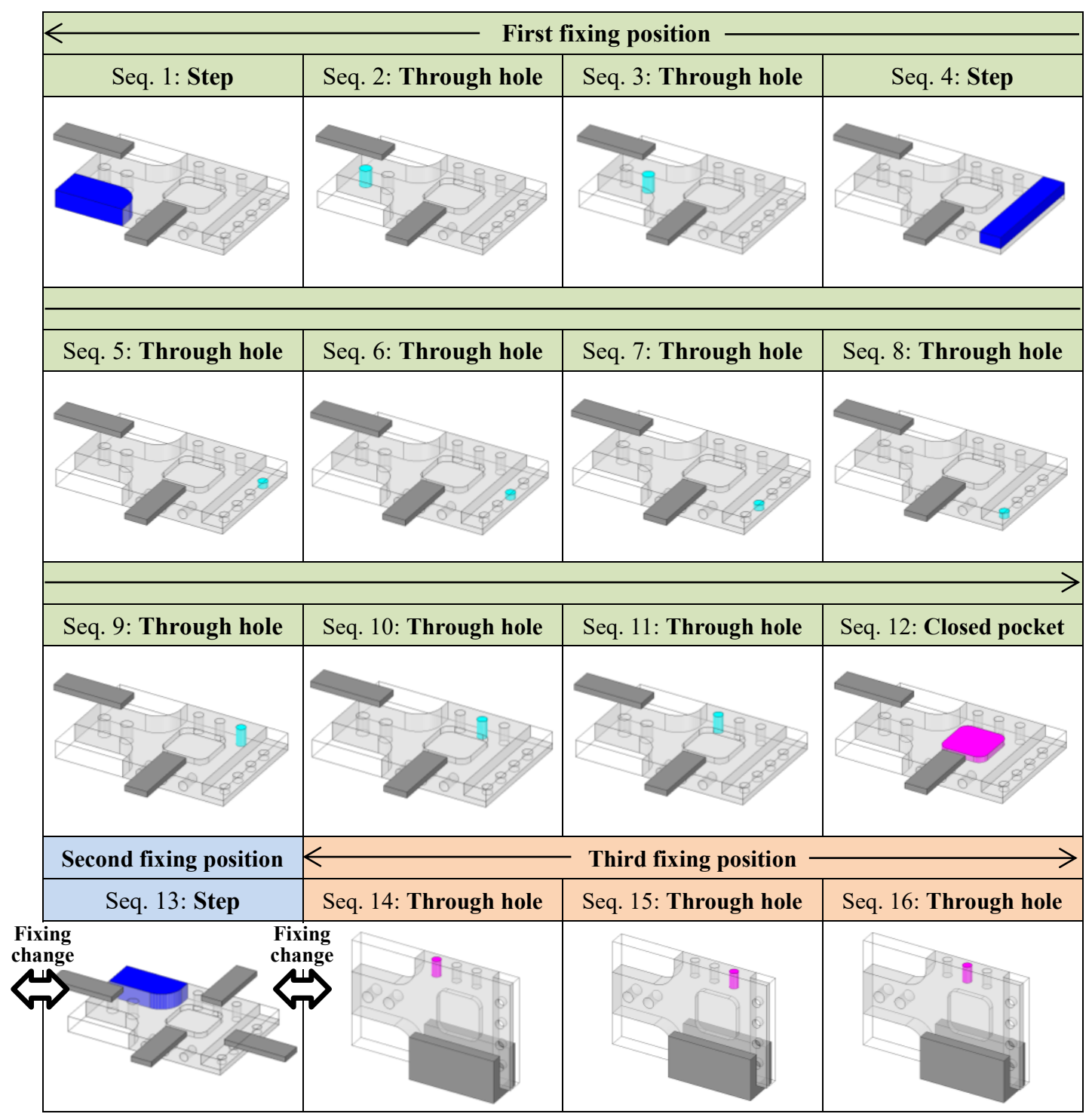

Fig. 21 Decided fixing position and recognized machining features in clamp-preceding method

\section{Conclusion}

In this study, a CAPP system for parts machining is developed considering the use of workholding devices. Two general workholding devices, a machine vise and strap clamps, are used to fix the workpiece for parts machining on a three-axis control machining center. In this system, CAD models of target shape and workpiece shape, as well as the specifications of workholding devices and toolings are first inputted. Then, appropriate workpiece fixing positions are calculated according to the workholding device being used. Machining features are recognized through allocating the machining sequence automatically, and the machining process parameters are finally determined by following the author's previously proposed method.

Case studies conducted to confirm the usefulness of the developed CAPP system found that fixing positions are obtained properly for various workpieces and workholding devices. Furthermore, it is recognized that users of the developed CAPP system can obtain useful information such as the number of fixing changes and machining times depending on the workholding devices being used. Thus, it is confirmed that the CAPP system has the potential to be effective by automatically determining machining process parameters of parts machining. 


\section{References}

Dwijayanti, K. and Aoyama, H., Basic study on process planning for turning-milling center based on machining feature recognition, Journal of Advanced Mechanical Design, Systems and Manufacturing, Vol. 8, No. 4 (2014), DOI: 10.1299/jamdsm.2014jamdsm0058.

El-Mehalawi, M. and Miller, R. A., A database system of mechanical components based on geometric and topological similarity. Part I: representation, Computer-Aided Design, Vol. 35, No. 1 (2003a), pp.83-94.

El-Mehalawi, M. and Miller, R. A., A database system of mechanical components based on geometric and topological similarity. Part II: indexing, retrieval, matching and similarity assessment, Computer-Aided Design, Vol. 35, No. 1 (2003b), pp.95-105.

Inoue, Y., and Nakamoto, K., Proposal of a recognition method of machining features in computer aided process planning system for complex parts machining, Transactions of the JSME (in Japanese), Vol. 83, No. 850 (2017), DOI: 10.1299/transjsme.16-00574.

Morinaga, E., Hara, T., Joko, H., Wakamatsu, H., and Arai, E., Improvement of computational efficiency in flexible computer-aided process planning, International Journal of Automation Technology, Vol. 8, No. 3 (2014), pp. 396405.

Nishida, I., Sato, R., and Shirase, K., Proposal of process planning system for end-milling operation considering product design constraints, The Institute of Systems, Control and Information Engineering, Vol. 30, No. 3 (2017), pp. 81-86 (in Japanese).

Nishida, I., Hirai, T., Sato, R., and Shirase, K., Automatic process planning system for end-milling operation considering CAM operator's intention, Transactions of the JSME (in Japanese), Vol. 84, No. 860 (2018), DOI: 10.1299/transjsme.17-00563.

Ratchev, S., Liu, S., Huang, W., and Becker, A.A., Milling error prediction and compensation in machining of low-rigidity parts, International Journal of Machine Tools and Manufacture, Vol. 44, No. 15 (2004), pp. 1629-1641.

Siebenaler, S., and Melkote, S., Prediction of workpiece deformation in a fixture system using the finite element method, International Journal of Machine Tools and Manufacture, Vol. 46, No. 1 (2006), pp. 51-58.

Teramoto, K., On-Machine estimation of workpiece deformation for thin-structured parts machining, Int. J of Automation Technology, Vol. 11, No. 6 (2017), pp. 978-983.

Ueno, A., and Nakamoto, K., Proposal of machining feature for CAPP system for multi-tasking machine tools, Transactions of the JSME (in Japanese), Vol. 81, No. 825 (2015), DOI: 10.1299/transjsme.15-00108.

Wang, L., Holm, M., and Adamson., G., Embedding a process plan in function blocks for adaptive machining, CIRP Annals, Vol. 59, No. 1 (2010), pp. 433-436. 